\title{
Vibrations in MagAO: Frequency-based analysis of on-sky data, resonance sources identification and future challenges in vibrations mitigation
}

\author{
Sebastián Zúñiga ${ }^{a^{*}}$, Javier Garcés ${ }^{a}$, Laird M. Close ${ }^{b}$, Jared R. Males ${ }^{b}$, Katie M. Morzinski ${ }^{b}$, \\ Pedro Escárate ${ }^{a}$, Mario Castro ${ }^{a}$, José Marchioni ${ }^{a}$ and Diego Zagals ${ }^{a}$ \\ ${ }^{a}$ AC3E, Departamento de Electrónica, Universidad Técnica Federico Santa María, Avenida \\ España 1680, Valparaíso, Chile \\ ${ }^{b} \mathrm{CAAO}$, Department of Astronomy and Steward Observatory, University of Arizona, Tucson \\ AZ 85721, USA
}

\begin{abstract}
Frequency-based analysis and comparisons of tip-tilt on-sky data registered with 6.5 Magellan Telescope Adaptive Optics (MagAO) system on April and Oct 2014 was performed. Twelve tests are conducted under different operation conditions in order to observe the influence of system instrumentation (such as fans, pumps and louvers). Vibration peaks can be detected, power spectral densities (PSDs) are presented to reveal their presence. Instrumentation-induced resonances, close-loop gain and future challenges in vibrations mitigation techniques are discussed.
\end{abstract}

Keywords: Adaptive Optics, vibration in AO control, frequency-based analysis, Magellan AO System

\section{INTRODUCTION}

Adaptive optics (AO) is a technology that compensates in real-time optical aberrations caused by atmospheric turbulence. Nowadays most of the large telescopes use this technology, one of these system is $\operatorname{MagAO}^{1}$ the 6.5m Magellan adaptive optics system. MagAO is the first adaptive secondary mirror based (ASM-based) AO system in the southern hemisphere, providing great diffraction-limited visible (0.6 - 1.0 micron) images down to 20 mas. $^{2}$ Despite these significant advances, other sources of perturbations still degrade the image quality, one of the most relevant are mechanical vibrations. Analysis of tip-tilt on-sky data is necessary in order to recognize the strongest vibrations components. Vibration peaks can be detected, power spectral densities (PSDs) are presented to reveal their presence. The main idea is to obtain information about the source of the vibrations and how this element(s) affect the tip and tilt modal amplitudes.

Instrumentation resonance and windshake noise is prejudicial for the performance of telescopes' adaptive optics systems. Thus, it is useful to analyze how the system's instrumentation is affecting the measured data. For this research, on-sky data taken with the MagAO system on October 31st, 2014 (FWHM of 0.8 [arcsec], mean wind speed of $22 \mathrm{MPH}$ ) at the Clay Telescope in LCO is analyzed and compared with data registered on April 17th, 2014. During this observing run, the system's elements, such as fans and pumps, are activated or deactivated in different configurations, collecting data from both the VisAO science camera and the waveform sensor (WFS).

The vibrations should be mitigated thanks to mechanical actions, but is highly desirable to develop new methods for measuring vibration in real time and also incorporate appropriate controllers in the adaptive optics systems in order to compensate this disturbance. Instrumentation-induced resonances, close-loop gain and future challenges in vibrations mitigation techniques are discussed.

(*) Corresponding author: sg.zunigaf@gmail.com 


\section{WORK OVERVIEW}

This work was conducted using closed-loop on-sky data taken during the observing runs of the University of Arizona MagAO Team, the nights of April 17th and October 31st, 2014, at the Clay Telescope in LCO, in order to study (under different operation conditions) the frequency-based information taken by sensors of the MagAO system. ${ }^{3}$

- From the April 17th run, one set of CCD camera data and one set of WFS data is used, in order to compare with the October data. These are "all-off" data, meaning only the necessary instrumentation is active.

- From the October 31th run, twelve pairs of CCD and WFS data are used. Each of these tests were held under different operation conditions ("test"), analyzing the influence on the frequency data from sensors of six elements within the system's instrumentation. The condition of these six elements during the twelve tests are described in Table 1. Between tests 4 and 5, the closed loop gain was re-optimized.

Since MagAO is able to obtain diffraction-limited visible (0.6 - 1.0 micron) images down to 20 mas, ${ }^{2}$ it is of great interest to detect factors that may affect the system's frequency response negatively. From each observation run, a different set of data was obtained from:

1. The VisAO science: $\mathrm{X}$ and $\mathrm{Y}$ movement of brightest pixel from scientific image detected. CCD-47 image position was measured at approx. 42 frames per second.

2. Waveform Sensor's (WFS) modal amplitudes, sampling nominally every 3 frames with a loop speed of $989.609 \mathrm{~Hz}$. Therefore, a sampling period of $0.0030315 \mathrm{sec}(1 / 3$ full loop speed) is obtained.

For each of the tests from the October data, and the set of measurements of the April data, Power Spectral Densities (PSD) are obtained, which is described in Section 3. The research done based on these data-sets can be classified in three main topics: comparing the "all-off" data from both observing runs, analyzing the effect of the closed-loop gain re-optimization, and identifying resonance sources by comparing the October tests.

\begin{tabular}{|l|l|l|l|l|l|l|}
\hline Test ID & Windscreen & visAO fans & Baysides & Louver & Primary Pump & Primary Fan \\
\hline vib_test_001 & down & on & on & open & on & on \\
vib_test_002 & down & on & on & close & on & on \\
vib_test_003 & down & on & on & close & off & on \\
vib_test_004 & down & on & on & close & off & off \\
vib_test_005 & down & on & on & close & off & off \\
vib_test_006 & down & on & on & close & off & on \\
vib_test_007 & down & on & on & close & on & on \\
vib_test_008 & down & on & on & open & on & on \\
vib_test_009 & down & off & on & close & off & off \\
vib_test_010 & down & on & off & close & off & off \\
vib_test_011 & up & on & on & close & off & off \\
vib_test_012 & down & on & on & close & off & off \\
\hline
\end{tabular}

Table 1: Summary of vibrations test over different operating conditions taken in the night of Oct. 31st, 2014. 


\section{SPECTRAL ANALYSIS METHOD}

The CCD and WFS data available for each measurement set has around to minute length. To obtain the PSD from each of them, the PSD is computed using the Welch method, ${ }^{4}$ cutting the data set into subset of 10 seconds, windowed with rectangular window without overlapping.

In order to obtain the normalized PSDs from the WFS data, the PSDs from the CCD camera data is utilized. Since it is possible to associate the scientific image's pixel length with the telescope's range in [mas], is it possible to normalize the CCD data. Then, the WFS tip and tilt PSDs were normalized using PSDs from the CCD camera data, reducing mean square error among both PSDs within the range of 15 to $21 \mathrm{~Hz}$ (lower frequencies are ignored since they are subject to aliasing, see Figure 1).
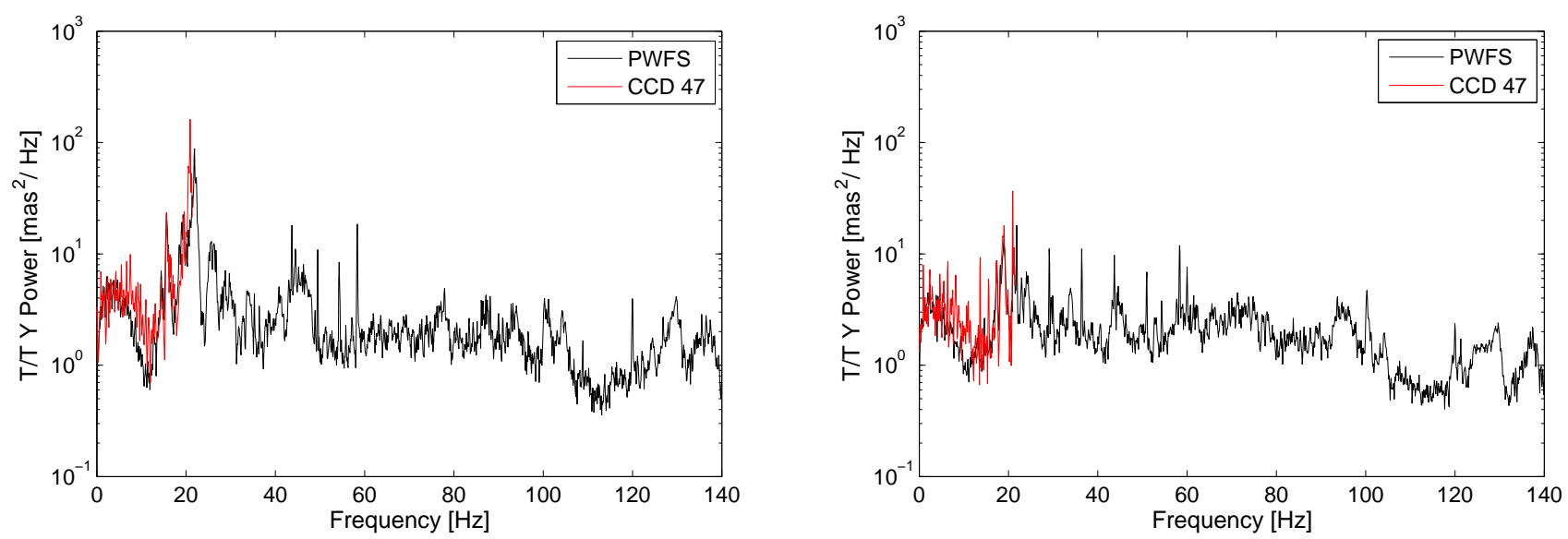

Figure 1: Vibration PSDs measured in $1 \mathrm{kHz}$ closed-loop on-sky. The PWFS tip/tilt PSDs (black) were normalized to the CCD-47 PSDs (red) from 15 to $21 \mathrm{~Hz}$.

\section{COMPARISON BETWEEN APRIL 17TH AND OCT 31ST DATA}

The data obtained from April 2014 and Oct 2014 were taken under different whether conditions. Better conditions were observed at $2014 \mathrm{~A}$ (seeing $\approx 0.6$ arcsec and mean wind speed $\approx 18 \mathrm{MPH}$ ) than $2014 \mathrm{~B}$ (seeing $\approx 0.8$ arcsec and mean wind speed $\approx 22 \mathrm{MPH}$ ). Given the PSDs plots obtained, it is possible to identify the main peaks induced by vibrations. It is evident to see that, regardless the weather conditions, the main resonance peaks in the PSDs match for each vibration test (see Figure 2).
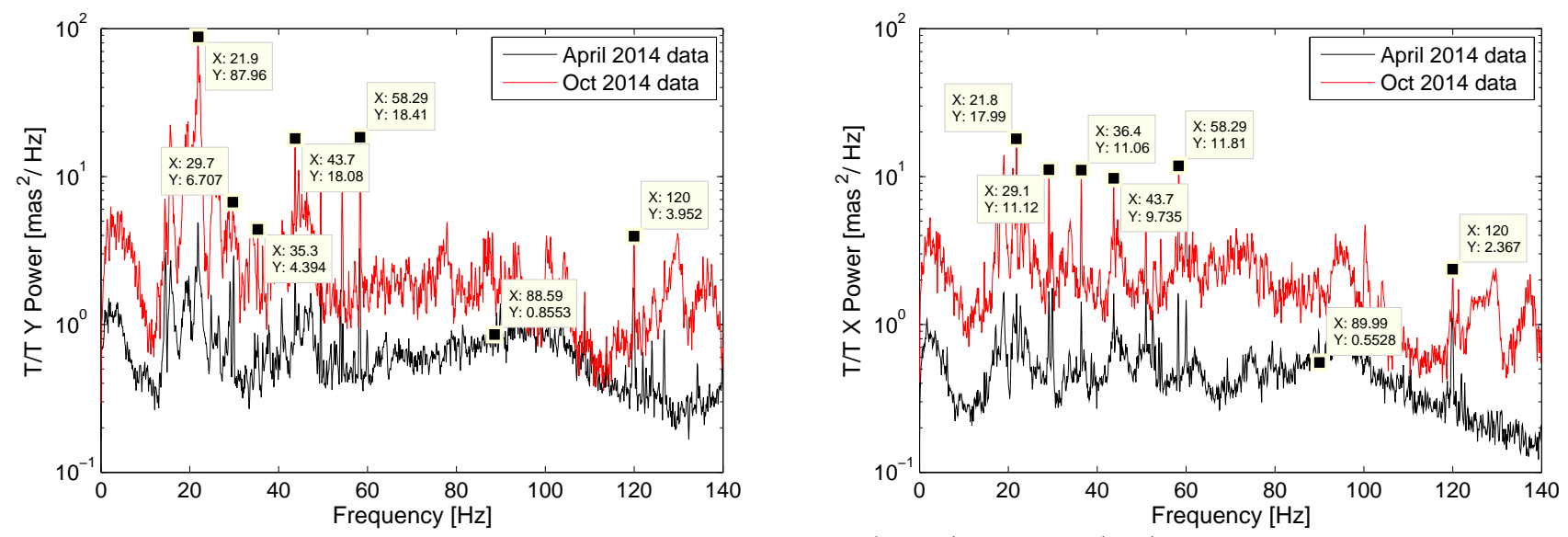

Figure 2: PSDs comparison between data registered on April (black) and Oct (red) 2014. Main resonance peaks are labeled. The estimated bandwidth is around $90 \mathrm{~Hz}$. 
Main resonance peaks were labeled (Figure 2). It presumed that peaks at $\sim 20 \mathrm{~Hz}$ and $\sim 60 \mathrm{~Hz}$ are induced by the telescope's inherent resonances and elements subject to the electrical grid's frequency respectively. Since some resonance frequency are half the other, probably these peaks may be the result of aliasing (Figure 2). The high frequency resonances (peaks at $120-140 \mathrm{~Hz}$ ) on Oct 2014 data can be assumed to be produced by vain ends/top ends high frequency vibrations given by the high tension over these structures. Finally, analyzing the frequency response of the close-loop data, the estimated bandwidth is around $90 \mathrm{~Hz}$ (Figure 2).

\section{CLOSED LOOP GAIN}

Comparison between vib test 01 and vib test 08 (see Table 1). The re-optimized gain PSD has better response in lower frequencies. It is presumed that the re-optimized gain produces a more gradual actuation which results in a better response at low frequencies (see Fig. 3).
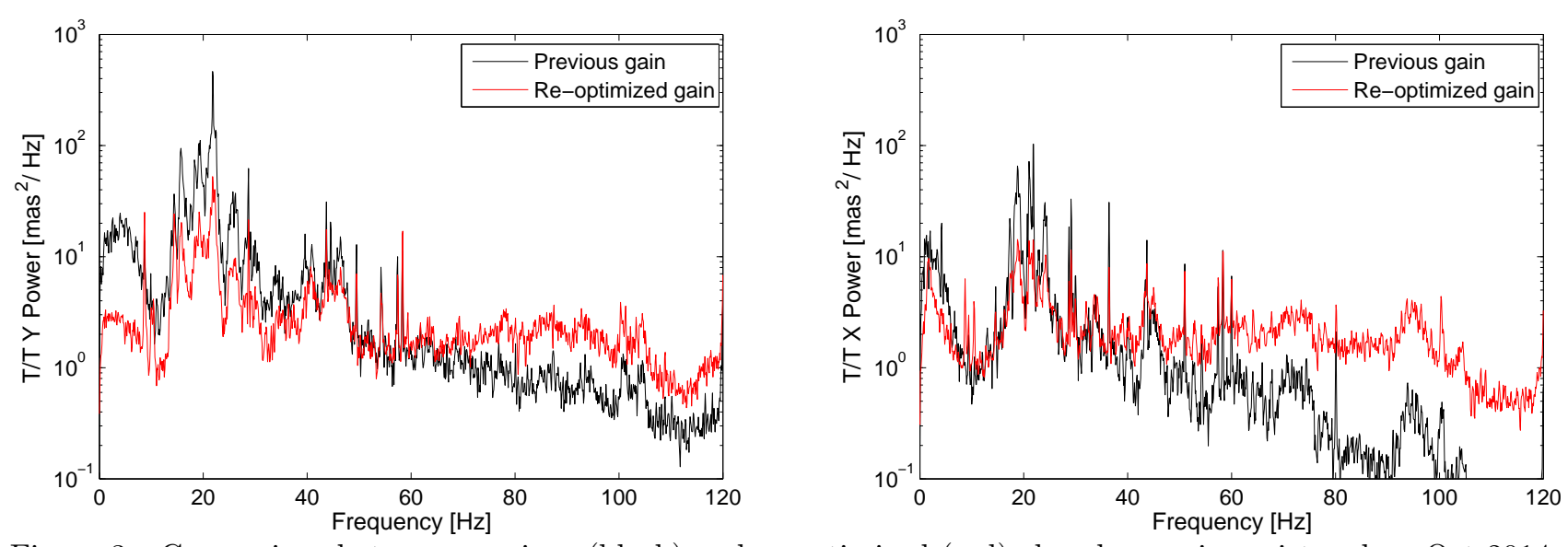

Figure 3: Comparison between previous (black) and re-optimized (red) close loop gain registered on Oct 2014.

A new frequency component can be seen around $10 \mathrm{~Hz}$ on the re-optimized close loop gain PSD (see Fig. 3). The re-optimized gain measurements was performed in a strong wind conditions (24 -25 MPH), therefore it can be assumed this new frequency component corresponds to wind-induced resonance.

\section{RESONANCE SOURCES IDENTIFICATION}

The main purpose of this section is show, in general terms, a method that can be useful to recognize vibration source in AO control. Since the WFS PSDs are normalized, it is possible to acquire numerical results of these influences by integrating both PSDs (with the element active and inactive), and obtaining the corresponding frequency's amplitude increase. In that regard twelve tests are conducted under different operation conditions (see Table 1). Six of these tests (tests 5 through 10) serve the purpose of analyzing the system's instrumentation. Table 2 describes the system state for each of these six tests. Since both modes 0 and 1 (tip and tilt) of WFS data is analyzed, twelve PSD plots are obtained, six for each mode.

\begin{tabular}{llllll}
\hline & Primary Fans & Primary Pumps & Louver & VisAO Fans & Baysides \\
\hline Test A & Off & Off & Closed & On & On \\
Test B & On & Off & Closed & On & On \\
Test C & On & On & Closed & On & On \\
Test D & On & On & Open & On & On \\
Test E & Off & Off & Closed & Off & On \\
Test F & Off & Off & Closed & On & Off \\
\hline
\end{tabular}

Table 2: System element's status during each test. Colors corresponds to curves in Figure 4. 
Tests A through $\mathrm{F}$ are plotted bottom to top respectively, with colors corresponding to Table 2. Tests B through $\mathrm{F}$ are displaced in powers of 10 along the vertical axis for comparison purposes. In Figure 4, resonance peaks across every test's PSD can be observed. These are not originated only by instrumentation elements, but by different factors, like the telescope's inherent resonances (peaks at $\sim 20 \mathrm{~Hz}$ ) and elements subject to the electrical grid's frequency (at $\sim 60 \mathrm{~Hz}$, and also including $\sim 30 \mathrm{~Hz}$ and $\sim 120 \mathrm{~Hz}$ peaks).
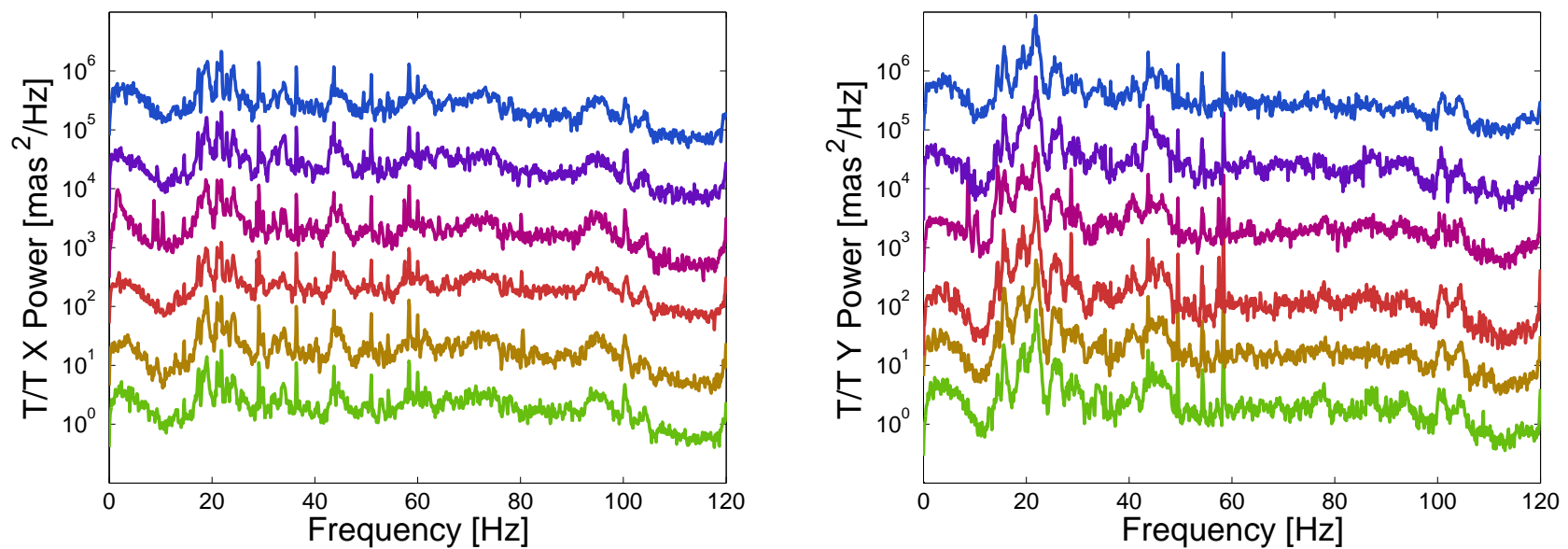

Figure 4: Vibration PSDs measured in $1 \mathrm{kHz}$ closed-loop on-sky, for Modes 0 and 1 of WFS data and for Tests A through F (Table 2).

Given that the Power spectral densities (PSDs) of recorded data are obtained, influences of the system's elements on sensors' frequency response can be identified. Using Test A's Y Power as example, it is possible to obtain a root-integrated-power value of 10.68 [mas] for a vicinity of $20 \mathrm{~Hz}$, and a value of 5.23 [mas] for vicinities of 30, 60 and $120 \mathrm{~Hz}$. These inherent resonances are significant in relation to the total root-integrated-power value of 20.98 [mas] RMS for this test. For more details and further frequency-based analysis of characteristics vibration of the MAgAO system instrumentation (such as fans, pumps and louvers), the reader is referred to Javier Garcés et al. ${ }^{5}$

\section{CONCLUSION AND FUTURE CHALLENGES}

Frequency-based analysis of MagAO tip-tilt on-sky data was performed. Power spectral densities (PSDs) plot was presented to reveal instrumentation-induced resonances on MagAO sensors. Through comparison between different weather condition data, it was possible to evidence inherent vibration of the telescope.

In the future, with the strong performance capabilities of the MagAO system, the resonance frequencies that may be considered to have a low impact on the sensors' response could have a greater impact for the next generation challenges of the system such detection of exoplanets close to bright stars or extremely faint ones. In that regard, real time PSDs estimation is suggested. The main goal of these approach is turn on and off different elements of the system and visualize in real time the effect on the PSDs plot. As it was observed, over a range of seeing and wind velocities, resonances are incredible stable. This result suggest that the mitigation of these vibrations components should be achieved with advanced controllers and system identification techniques, such as LQG, $\mathrm{H}_{\infty}$ or model predictive control (MPC).

\section{ACKNOWLEDGMENTS}

The research for this paper couldn't have been possible without the support and generosity of the MagAO Team: Dr. Laird Close, Dr. Jared Males and Dr. Katie Morzinski, who have allow us to work and investigate the MagAO system alongside them during the Winter Research Program 2015 at the University of Arizona.

I gratefully acknowledge the support of the Advanced Center for Electrical and Electronic Engineering (AC3E) Basal Project FB0008 and the Universidad Técnica Federico Santa María, as well as the financial support from CONICYT, Chile. 


\section{REFERENCES}

[1] K. M. Morzinski, L. M. Close, J. R. Males, D. Kopon, P. M. Hinz, S. Esposito, A. Riccardi, A. Puglisi, E. Pinna, R. Briguglio, M. Xompero, F. Quirós-Pacheco, V. Bailey, K. B. Follette, T. J. Rodigas, Y.-L. Wu, C. Arcidiacono, J. Argomedo, L. Busoni, T. Hare, A. Uomoto, and A. Weinberger, "MagAO: Status and on-sky performance of the Magellan adaptive optics system," Proc. SPIE 9148, Adaptive Optics Systems IV, 914804 (July 21, 2014).

[2] L. M. Close, J. R. Males, K. Morzinski, D. Kopon, K. Follette, T. J. Rodigas, P. Hinz, Y.-L. Wu, A. Puglisi, S. Esposito, A. Riccardi, E. Pinna, M. Xompero, R. Briguglio, A. Uomoto, and T. Hare, "Diffraction-limited visible light images of Orion Trapezium Cluster with the Magellan Adaptive Secondary Adaptive Optics System (MagAO)," The Astrophysical Journal, vol. 774, no. 2, p. 94, Aug. 2013.

[3] J. R. Males, L. M. Close, O. Guyon, K. Morzinski, A. Puglisi, P. Hinz, K. B. Follette, J. D. Monnier, V. Tolls, T. J. Rodigas, A. Weinberger, A. Boss, D. Kopon, Y. Wu, S. Esposito, A. Riccardi, M. Xompero, R. Briguglio, and E. Pinna, "Direct imaging of exoplanets in the habitable zone with adaptive optics," Proc. SPIE 9148, Adaptive Optics Systems IV, 914820 (July 21, 2014).

[4] P. Welch, "The use of fast Fourier transform for the estimation of power spectra: A method based on time averaging over short, modified periodograms," IEEE Trans. Audio Electroacoust., vol. 15, no. 2, pp. 70-73, Jun. 1967.

[5] J. Garcés, S. Zuñiga, L. M. Close, J. R. Males, K. M. Morzinski, P. Escárate, M. Castro, J. Marchioni, and D. Rojas, "Vibrations in MagAO: Resonance sources identification and first approaches for modeling and control," Proc. SPIE Astronomical Telescopes + Instrumentation, Adaptive Optics System V, (July, 2016). 